\title{
Human Activity Recognition through Smartphone's Tri-Axial Accelerometer using Time Domain Wave Analysis and Machine Learning
}

\author{
Sarthak Gupta \\ Guru Tegh Bahadur Institute of Technology, \\ GGSIPU, New Delhi, India
}

\author{
Ajeet Kumar \\ Maharaja Surajmal Institute of Technology, \\ GGSIPU, New Delhi, India
}

\begin{abstract}
Human activity recognition aims at capturing current state of human in the immediate environment. This article poses a novel approach to predict the human activity by exploiting the data collected from smartphone's triaxial accelerometer sensor. This approach employs a time domain wave analysis on the collected data and extracts relevant features that intuitively distinguish various activities such as walking, standing, running, jogging and sitting. The method then classifies the extracted features using various Machine learning algorithms comprising of SVM, J48 and AdaBoost and Random Forest. This work attains a steadfast accuracy of $\mathbf{9 8 . 8 2 8 3 \%}$. HAR has numerous uses, ranging from healthcare, HCI, ubiquitous networks, to entertainment. The need for ever-increasing accuracy levels is inevitable and this work serves the purpose.
\end{abstract}

\section{General Terms}

Data mining, classification algorithms, WEKA tool, Activity recognition, Machine learning.

\section{Keywords}

AdaBoost, J48, Support Vector Machines, Machine Learning, Activity Recognition, Random Forests, WEKA, Machine learning.

\section{INTRODUCTION}

Mobile devices and specifically smartphones have recently begun to employ powerful and diverse sensors. These sensors include Accelerometer, Gyroscope, Light, Battery, Temperature, Magnetometer, and many more, each with their specific function. Because of advancement in technologies, these smartphones are becoming more compact, user-friendly, and most importantly, substantially fast computers. The ability to send, receive data have made them ubiquitously available in our society. These devices, by virtue of inherent computing ability and powerful sensors, have introduced a new research area - Wireless Sensor Data mining. Sensors can be used to capture data pertaining to battery of device, to forces being applied on the device. One such sensor capability has been exploited in this paper, that is, the accelerometer sensor.

Accelerometer sensor measures the acceleration forces being applied on mobile device, with respect to three dimensional $\mathrm{X}, \mathrm{Y}$ and $\mathrm{Z}$ coordinate system. The sensor measures the forces in $\mathrm{m} / \mathrm{s}^{2}$. This paper uses all this knowledge to classify the human activity under 4 categories, that is, Walking, Running, Sitting and Standing. We tend to exploit the fact that, while the user is performing any activity, the smartphone kept in the jeans pocket would experience a force applied (on $\mathrm{Y}$ axis) that represents activity pattern. This pattern can be distinguished for different activities.

The Authors have chosen Android-based cell phones as the platform for our WISDM project because the Android operating system is free, open-source, easy to program, and expected to become a dominant entry in the cell phone marketplace (this is clearly happening) [5]. This project can also be scaled to other mobile platforms such as IOS and others in market. Accelerometers were initially included in these devices to support advanced game play and to enable automatic screen rotation but they clearly have many other applications. [5] In fact, there are many useful applications that can be built if accelerometers can be used to recognize a user's activity. For example, we can automatically monitor a user's activity level and generate daily, weekly, and monthly activity reports, which could be automatically emailed to the user [5]. These reports would indicate an overall activity level, which could be used to gauge if the user is getting an adequate amount of exercise and estimate the number of daily calories expended. These reports could be used to encourage healthy practices and might alert some users to how sedentary they or their children actually are.

In order to recognize the activity through smartphone using supervised learning, the authors first collect the data while user performs the activity with an android based application gathering the required data. Once the dataset is constructed, we employ different machine learning algorithms to classify the activity under the aforementioned categories. Following is the organization of rest of the paper:-

Section 2 describes about literature Survey; the researches that have been performed in this area. Section 3 elucidates the methodology and model proposed in this research. Section 4 and 5 summarizes the results of the research. Section 6 throws light on any future work scope that can augment this research. Concluding the paper with Section 7, it acknowledges the work of various researches that have been conducive in concocting this article.

\section{LITERATURE SURVEY}

The area of activity recognition is not new. Amin Rasekh et al. designed [1] a activity recognition system based on a smartphone. The system uses a3-dimentional smartphone accelerometer as the only sensor to collect time series signals, from which 31 features are generated in both time and frequency domain. Activities are classified using 4 different passive learning methods, i.e., quadratic classifier, k-nearest neighbor algorithm, support vector machine, and artificial neural networks. Results show that the classification rate of passive learning reaches $84.4 \%$.Akram Bayat et al. [2] propose a recognition system in which a new digital low-pass 
filter is designed in order to isolate the component of gravity acceleration from that of body acceleration in the raw data. The system was trained and tested in an experiment with multiple human subjects in real-world conditions. It was found that using the average of probabilities as the fusion method reached an overall accuracy rate of $91.15 \%$. Sang and $\mathrm{Vu}$ Ngoc Thanh [3] represented daily activity monitoring using accelerometer and gyroscope sensors embedded in a smartphone. Signals were recorded from accelerometer and gyroscope sensors while a user was wearing the smartphone and performing different activities. For offline analysis, the classification algorithms with k-nearest-neighbor $(\mathrm{kNN})$, artificial neural network (ANN) and support vector machine (SVM) were applied to recognize user's activities. The overall accuracy of recognizing five activities was $74 \%$ for $\mathrm{kNN}$, $75.3 \%$ for ANN and $94.5 \%$ for SVM respectively. Rao Fu et al. [4] focus on improving classification accuracy and reducing computational complexity for human activity recognition problem on public datasets. Firstly they obtained raw data from sensors. In the case of smartphone, the data was acquired from the accelerometer. This raw data preprocessed for classification training algorithm. For smartphone accelerometer, time series data were segmented into fractions. Features are then generated and selected based on raw data. After giving enough data samples, model was mapped using proper learning algorithms. The overall accuracy achieves over $80 \%$.

Our work is different in several aspects. One contribution the authors intend to make is that the data they collect and continue to in future as well, will be made public that can prove to be of valuable source to other researches. They also demonstrate how raw time series accelerometer data can be transformed into examples that can be used by conventional classification algorithms. The authors demonstrate that it is possible to perform activity recognition with commonly available (nearly ubiquitous) equipment and yet achieve highly accurate results. Finally, they believe that our work will help bring attention to the opportunities available for mining wireless sensor data and will stimulate additional work in this area. Moreover, the accuracy levels achieved are higher than any research available in the field.

\section{METHODOLOGY}

\subsection{Experimental Setup}

In order to build up a dataset for the supervised learning task, it was necessary to select enough users to carry a smartphone and record the data while performing certain activities. The data was collected using an android based application developed specifically to record the forces being applied on smartphone's coordinate system. 20 subjects were enlisted for this purpose-having weight in the range of 53 Kilograms to 98 Kilograms- who were given ASUS ZenFone 5 android device, installed with the android application. These subjects carried the android device in their jeans' pocket while performing the activities, with application running. They were asked to perform each activity for at least 4 minutes, so that the pattern of different activities can be recorded and any anomaly generated does not affect the accuracy. The forces being applied on the device can be visualized as a very common example - The forces they experience while sitting in a car. When a car accelerates, decelerates or take a sharp turn, a person experience a force as explained in Newton's First Law of Inertia - "An object at rest will remain at rest unless acted on by an unbalanced force. An object continues in motion with the same speed and in the same direction unless acted upon by an unbalanced force." The pictures below explain the concept.

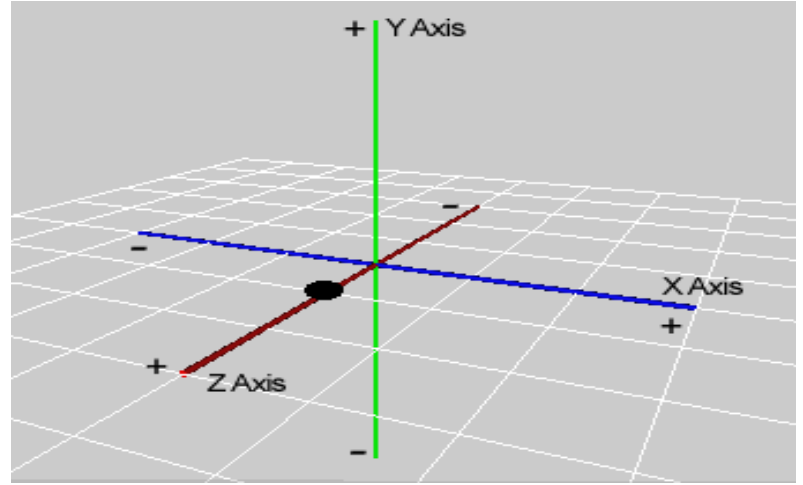

Fig 1: Normal Acceleration

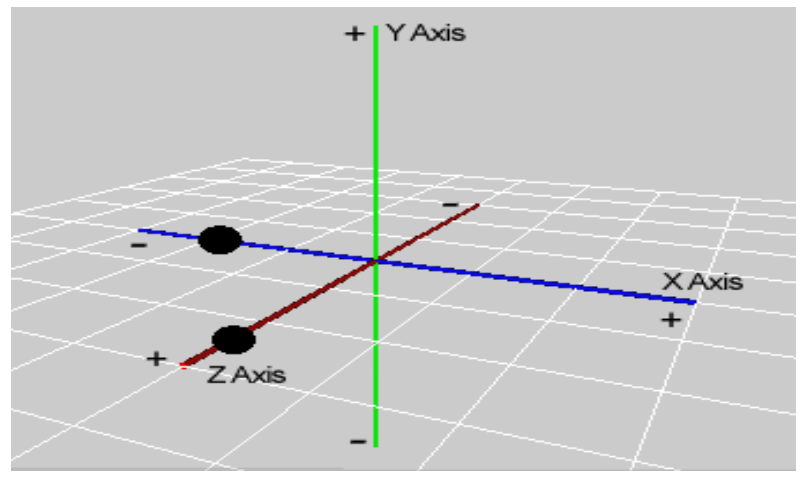

Fig 2: High acceleration on right turn

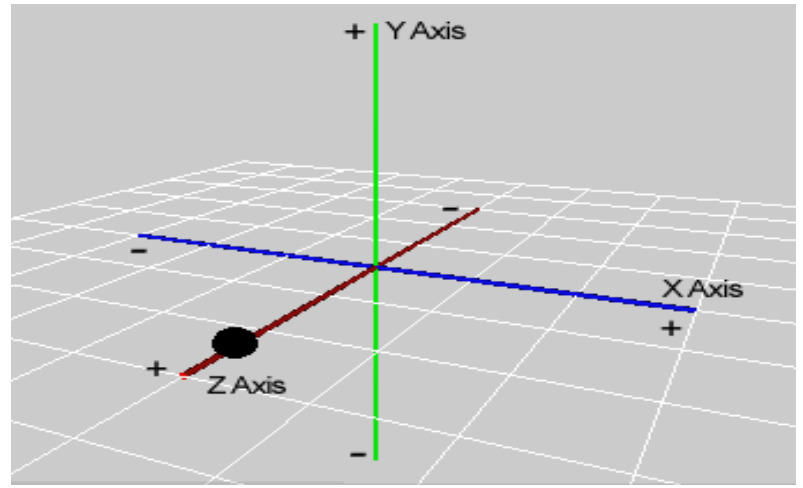

Fig 3: Sharp Acceleration.

The black dots represent the forces on a body under mentioned scenarios.

The android application developed was specifically designed to capture the smartphone's movement while subject was performing the activity. It recorded the forces being applied on Smartphone along X, Y, and $\mathrm{Z}$ axis in terms of $\mathrm{m} / \mathrm{s}^{2}$. The application used various smartphone sensors that are ubiquitously found in smartphones. These sensors consist of accelerometer, gyroscope, GPS and others. The application uses accelerometer and records the data in terms of acceleration being applied on the device. The data was collected at a frequency of 0.5 seconds. Hence, 120 samples were collected every minute. The update interval of the sensor was set to "very fast". This setting is employed while developing the android application, which allows developer to set the update interval of various sensors. The update interval defines a rate at which sensors are updated with the new value. Below are some snapshots of the Android application. 


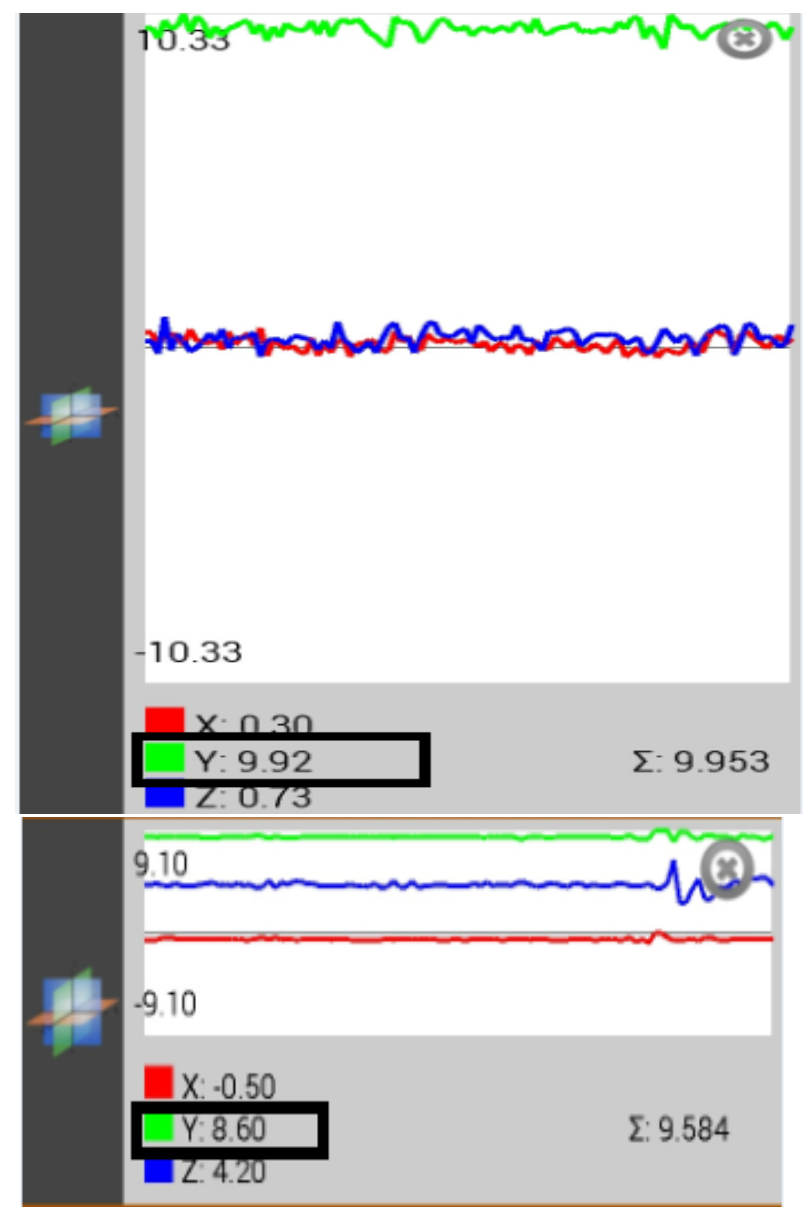

Fig 4: Android based application

\subsection{Feature Extraction}

Standard classification algorithms cannot be applied directly to raw time series data. Therefore, it is necessary to extract some relevant features that can differentiate various human activities. Following features are extracted for classification purpose.

\section{Table 1: Features Extracted}

\begin{tabular}{|l|l|}
\hline Average Peak value & $\begin{array}{l}\text { Mean value of peaks } \\
\text { observed in time-series data }\end{array}$ \\
\hline Average Peak rising time & $\begin{array}{l}\text { Time required per sample to } \\
\text { reach to its maxima }\end{array}$ \\
\hline Average Peak fall time & $\begin{array}{l}\text { Time required per sample to } \\
\text { reach to its minima }\end{array}$ \\
\hline Average Time per sample & Time span for each sample \\
\hline Average Time between peaks & $\begin{array}{l}\text { Time between each peak } \\
\text { value }\end{array}$ \\
\hline
\end{tabular}

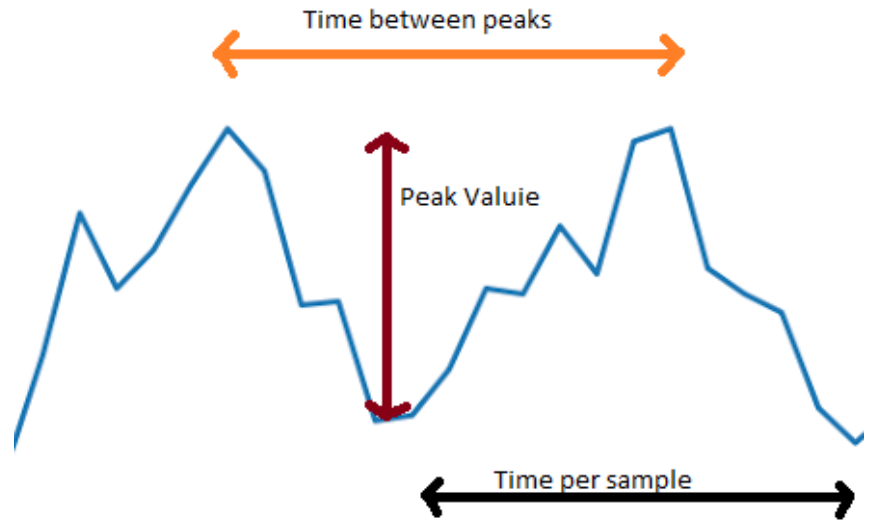

Fig 5: Description of Features: - Time between peaks, Peak value and Time per sample

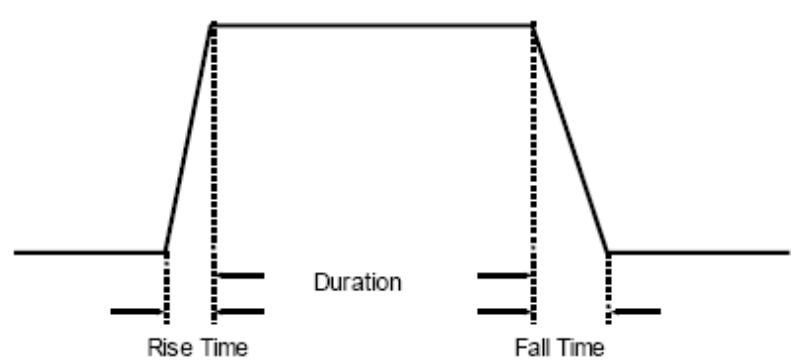

Fig 6: Rise time and Fall time of peak

Time-series data collected from Mobile's accelerometer sensor was analyzed in MATLAB to extract aforementioned features. Time-series data pertaining to different activities is manifested in following section. The graphs have been visualized using TABLEAU public 9, a data visualization tool.

\subsection{Activities}

Subjects were asked to perform activities in their usual manner for 4 minutes each, keeping the device in their jeans pocket for sitting and standing, walking and running, with the android application turned on. It can be seen that time-series data can be used to distinguish various activities. The acceleration on device's $\mathrm{Y}$ axis is measured along $\mathrm{Y}$ axis and $\mathrm{X}$ axis represents time interval of 0.5 seconds.

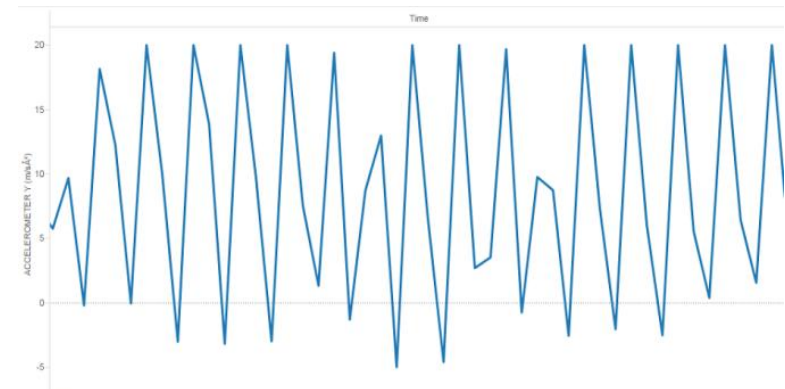

Fig 7: Time series data for Running 


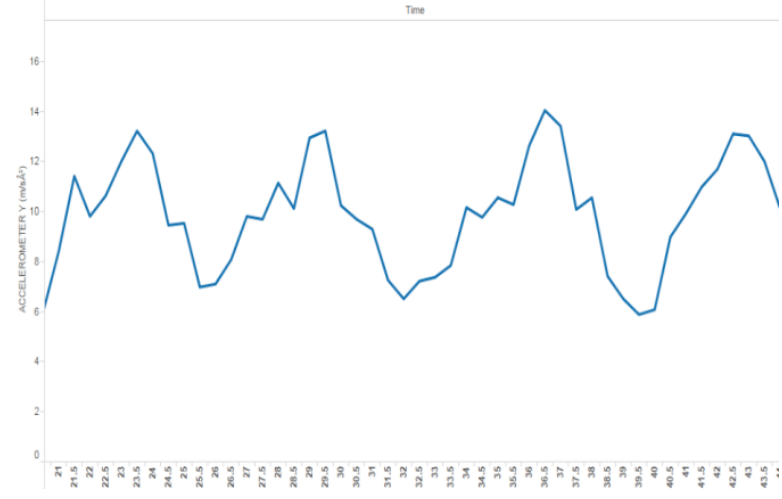

Fig 8: Time series data for Walking

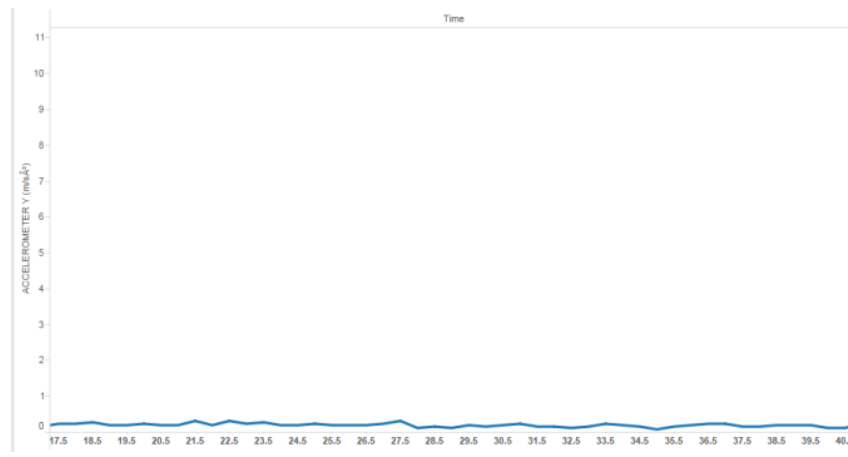

Fig 9: Time series data for Sitting

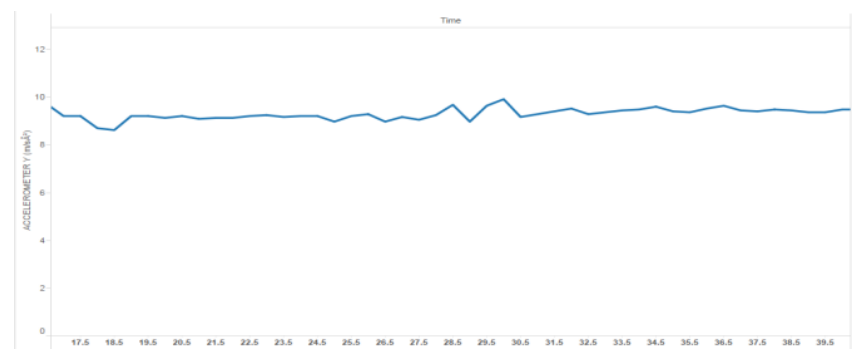

Fig 10: Time series data for Standing

\subsection{Classification}

Once dataset was prepared, machine learning algorithms were applied to classify the activity under four categories. Some activities had fewer examples, mainly because they require long time and fewer data for them sufficed the requirements. WEKA was used to apply the algorithms under default settings. They employed J48, Support vector machines, AdaBoost and Random Forest on the dataset. Out of these, AdaBoost showed best accuracy levels.

\subsubsection{AdaBoost}

The AdaBoost algorithm proposed by Yoav Freund and Robert Schapire is one of the most important ensemble methods, since it has solid theoretical foundation, very accurate prediction, great simplicity (Schapire said it needsonly "just 10 lines of code"), and wide and successful applications [6]. XindongWu et al. in paper [26] has described AdaBoost algorithm as follows:-

Let $X$ denote the instance space and $Y$ the set of class labels. Assume $Y=\{-1,+1\}$. Given a weak or base learning algorithm and a training set $\left\{\left(x_{1}, y_{1}\right),\left(x_{2}, y_{2}\right), \ldots,\left(x_{m}, y_{m}\right)\right\}$ where $x_{i} \in X$ and $y_{i} \in Y \quad(i=1, \ldots, m)$, the AdaBoost algorithm works as follows. First, it assigns equal weights to all the training examples $\left(\boldsymbol{x}_{i}, y_{i}\right)(i \in\{1, \ldots, m\})$. Denote the distribution of the weights at the $t$-th learning round as $D t$. From the training set and $D t$ the algorithm generates a weak or base learner $h t: X \rightarrow Y$ by calling the base learning algorithm. Then, it uses the training examples to test $h t$, and the weights of the incorrectly classified examples will be increased. Thus, an updated weight distribution $D t+1$ is obtained. From the training set and $D t+1$ AdaBoost generates another weak learner by calling the base learning algorithm again. Such a process is repeated for $T$ rounds, and the final model is derived by weighted majority voting of the $T$ weak learners, where the weights of the learners are determined during the training process. The pseudo code for the algorithm is presented in [6] as:-

Input: $\quad$ Data set $\mathrm{D}=\left\{\left(x_{1}, y_{1}\right),\left(x_{2}, y_{2}\right), \ldots,\left(x_{m}, y_{m}\right)\right\}$;

Base learning algorithm $\boldsymbol{L}$;

Number of learning rounds $\boldsymbol{T}$.

\section{Process:}

$D_{1}(i)=1 / m \quad \%$ Initialize the weight Distribution

$h_{t}=L\left(D, D_{t}\right) ; \%$ Train a weak learner $h_{t}$ from D usingdistribution $\mathrm{D}_{\mathrm{t}}$ $\varepsilon_{t}=\operatorname{Pr}_{i \sim D_{i}}\left[h_{t}\left(x_{i} \neq y_{i}\right)\right] ; \%$ Measuretheerror of $h_{t}$ $\alpha_{t}=\frac{1}{2} \ln \left(\frac{1-\varepsilon_{t}}{\varepsilon_{t}}\right) ; \%$ Determinethe weightof $h_{t}$ $D_{t+1}(i)=\frac{D_{t(i)}}{Z_{t}} \times\left\{\begin{array}{ll}\exp \left(-\alpha_{t}\right) & \text { if } h_{t}\left(x_{i}\right)=y_{i} \\ \exp \left(\alpha_{t}\right) & \text { if } h_{t}\left(x_{i}\right) \neq y_{i}\end{array}\right\}$ $D_{t+1}(i)=\frac{D_{t}(i) \exp \left(-\alpha_{t} y_{i} h_{t}\left(x_{i}\right)\right)}{Z_{t}}$

$D_{1}(i)=1 / m \quad \%$ Initialize the weight Distribution

Output: $H(x)=\operatorname{sign}\left(\sum_{t=1}^{T} \alpha_{t} h_{t}(x)\right)$

End

\section{RESULTS}

Several Classification algorithms were used to monitor the accuracy levels. The results are summarized in table 2 .

Table: 2 Summarized results

\begin{tabular}{|l|l|l|}
\hline S.no. & Classifier & Accuracy \\
\hline 1 & AdaBoost & $\mathbf{9 8 . 8 2 8 3 \%}$ \\
\hline 2 & J48 & $\mathbf{9 6 . 7 4 7 5 \%}$ \\
\hline 3 & Support Vector Machines & $\mathbf{9 5 . 7 4 7 5 \%}$ \\
\hline 4 & Random Forest & $\mathbf{9 3 . 7 4 7 5 \%}$ \\
\hline
\end{tabular}




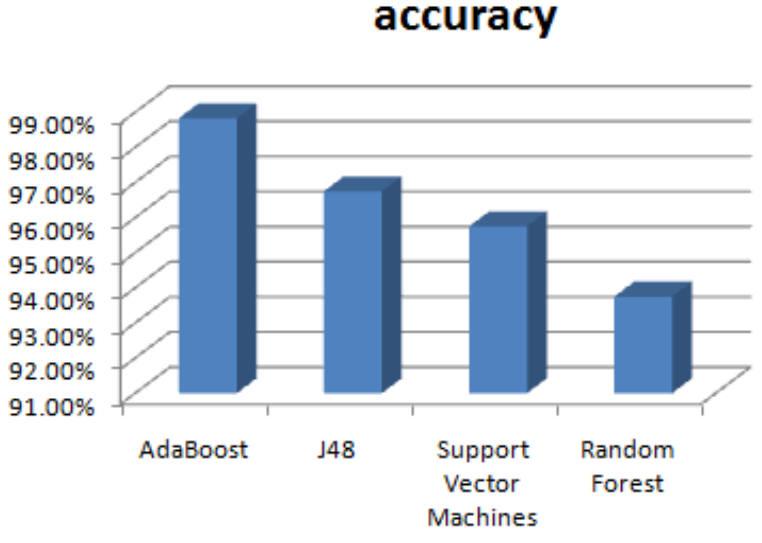

Fig 11: Performance Comparison

\section{CONCLUSION}

In this paper the authors described how a smart phone can be used to perform activity recognition, simply by keeping it in one's pocket. They further showed that activity recognition can be highly accurate, with most activities being recognized correctly over $90 \%$ of the time. In addition, these activities can be recognized quickly, since each example is generated from only 10 seconds worth of data.The authors have several interesting applications in mind for activity recognition and plan to implement some of these applications in the near future.

\section{FUTURE WORK}

The authors intend to improve our research in several ways. First, they will try to recognize additional activities such as Jogging, Climbing stairs up and down, bicycling, driving and many more. Second, they would collect data from more people in the hope that it will further improve our results. Third, they will try to evaluate performance in different positions where phone will be kept in shirt pocket, belt loop. Fourth, they will try to enlarge the scale so that the system can be employed in real time where the classification result would be reported back to user. As evident, there is always a room for improvement. The authors will try to improve our accuracy and reach higher levels with more sophisticated system. The authors would also try to push all the data to central server, where data from different devices would be gathered onto a single server and accuracy can further be improved where data from various recorded activities can be mined pertaining to different users.

\section{REFERENCES}

[1] Amin Rasekh, Chien-An Chen Yan Lu, Human Activity Recognition using Smartphone, Texas A\&M University

[2] Akram Bayat*, Marc Pomplun, Duc A. Tran Department of Computer Science, A Study on Human Activity Recognition Using Accelerometer Data from Smartphones, Akram Bayat*, Marc Pomplun, Duc A. Tran Department of Computer Science, University of

[3] Vu Ngoc Thanh Sang, Nguyen Duc Thang, Vo Van ToiAffiliated withDepartment of Biomedical Engineering, International University - VNU, Nguyen Duc Hoang, Truong Quang Dang Khoa, Human Activity Recognition and Monitoring Using Smartphones, 5th International Conference on Biomedical Engineering in VietnamVolume 46 of the series IFMBE Proceedings pp 481-485

[4] Rao Fu, Yao Song Weipu Zhao Computing Science Simon Fraser University weipuz@sfu.caFröhlich, B. and Plate, J. 2000. The cubic mouse: a new device for threedimensional input. In Proceedings of the SIGCHI Conference on Human Factors in Computing Systems

[5] Jennifer R. Kwapisz, Gary M. Weiss, Samuel A. Moore, Activity recognition using cell phone accelerometers, ACM SIGKDD Explorations Newsletter Volume 12 Issue 2, December 2010 Pages 74-82.

[6] XindongWu et al., 2008, Top 10 algorithms in data mining, Springer, KnowlInfSyst (2008) 14:1-37

[7] Brezmes, T., Gorricho, J.L., and Cotrina, J. 2009. Activity Recognition from accelerometer data on mobile phones In IWANN '09: Proceedings of the 10th International Work- Conference on Artificial Neural Networks, 796-799. 